\title{
Cost variation analysis of commonly prescribed anti-diabetic drugs available in Indian market: a pharmaco-economic study
}

\author{
Kajal S. Gupta, Milind L. Pardeshi*, Rajesh S. Hiray
}

Department of Pharmacology, B. J. Government Medical College and Sassoon General Hospital, Pune, Maharashtra, India

Received: 14 November 2021

Accepted: 03 December 2021

*Correspondence:

Milind L. Pardeshi,

Email: drmilindpardeshi@gmail.com

Copyright: $@$ the author(s), publisher and licensee Medip Academy. This is an open-access article distributed under the terms of the Creative Commons Attribution Non-Commercial License, which permits unrestricted non-commercial use, distribution, and reproduction in any medium, provided the original work is properly cited.

\begin{abstract}
Background: Diabetes mellitus (DM) is a chronic metabolic disorder requiring lifelong treatment. Due to rapid expansion of urbanization, unhealthy diet habits and sedentary lifestyle, the incidence of DM is increasing. The chronic nature of DM causes significant personal suffering and economic difficulty to families. The was aimed at investigating the cost difference in various brands of the same oral anti-diabetic drug.

Methods: The minimum and the maximum cost in rupees (INR) of a particular anti-diabetic drug manufactured by various pharmaceutical companies were obtained from current index of medical specialties (CIMS) website, Indian drug review (IDR) 2021 issue and National pharmaceutical pricing authority-pharma sahi daam. The cost ratio and percentage cost variation were noted for each brand.

Results: Amongst single drug therapy, metformin $500 \mathrm{mg}$ sustained release showed highest price variation $(3668 \%)$. Minimum cost variation was found with glipizide $2.5 \mathrm{mg}(65 \%)$. Amongst the fixed dose combinations, highest cost variation was seen with glimepiride $2 \mathrm{mg}+$ metformin $1000 \mathrm{mg}(2703 \%)$ while minimum cost variation was found with repaglinide $1 \mathrm{mg}+$ voglibose $0.3 \mathrm{mg}(29 \%)$.

Conclusions: A noticeable cost variation was found in different brands of the same anti-diabetic drug. Prescribing a more expensive brand when a cheaper one is available can burden the patient financially and thus reduce patient compliance. In addition, the Government should also include more anti-diabetic drugs under the price control policy to ensure that affordable and efficacious medicines are available to all.
\end{abstract}

Keywords: Anti-diabetic agents, Cost variation, Pharmaco-economics, Adherence, Brands

\section{INTRODUCTION}

DM is a chronic metabolic disorder requiring lifelong treatment. ${ }^{1}$ Due to rapid urbanization, unhealthy diet habits and a sedentary lifestyle, the incidence of DM is increasing. ${ }^{2}$ The global DM prevalence in 2019 was estimated to be $9.3 \%$ (463 million people) and is expected to rise to $10.2 \%$ (578 million) by 2030 and $10.9 \%$ (700 million) by $2045 .^{3}$ In India too, DM is fast gaining the status of a potential epidemic with nearly 65 million diabetic individuals currently diagnosed with the disease. ${ }^{4}$
The chronic nature of DM causes significant personal suffering and economic difficulties to families. ${ }^{5}$ Since patients with chronic illnesses often take multiple medications, they are particularly susceptible to medication cost pressures. Patients who are concerned about out-of-pocket medication costs often restrict their use of prescription drugs. ${ }^{6}$

Indian market is predominantly a branded generic market, more than one company sells a particular drug under different brand names apart from the innovator company. Hence, the number of pharmaceutical products available in 
the market is very high in the range of 60,000-70,000 products. This situation has led to greater price variation among drugs marketed. ${ }^{7}$ Studies suggested that the prescribing doctors have a poor knowledge of cost of drugs. Much more focus is required in the education of physicians about costs and the access to cost information so that they can choose the least costly medication when there are no substantial differences in safety and efficacy between the least and most expensive. ${ }^{8}$

Increasing pharmaceutical costs negatively impacts patients in two ways. First, high direct expenses for those of limited resources may mean a choice between medicines and necessities such as food or clothing. Alternatively, patients who do not take their medicine as directed or go without the potentially beneficial therapies suffer negative health consequences. ${ }^{9,10}$ Patients may also sometimes be too embarrassed to tell their physicians when they cannot afford their medicines. ${ }^{6}$

The aim of our study was to analyse cost variation of commonly prescribed oral anti-diabetic drugs which were available in the Indian market under various brand names. In addition, we have also compared their cost-ratio and percentage cost variation alongwith their maximum and minimum price.

\section{METHODS}

A cross-sectional, observational study was carried out at a tertiary hospital in Western India over 2 months from 1 May 2021 to 31 June 2021 after approval from the institutional ethics committee. The study adhered to the tenets of Declaration of Helsinki. Price in INR of commonly used oral anti-diabetic drugs of same preparation and same strength manufactured by different pharmaceutical companies was obtained from CIMS website, IDR 2021 issue and National pharmaceutical pricing authority-pharma sahi daam. The cost of 10 tablets (pack size) was calculated. The minimum and the maximum cost in INR of a particular anti-diabetic drug manufactured by various pharmaceutical companies of the same strength was noted. The cost ratio defined as the ratio of the costliest brand to that of the cheapest brand of the same drug was calculated as follows, ${ }^{11}$

Cost ratio $=\frac{\text { price of the costliest brand }}{\text { price of the least costly brand }}$.

Percentage cost variation was calculated as follows, ${ }^{12}$

Percentage cost variation $=\frac{\text { maximum } \cos t-\text { minimum } \operatorname{cost}}{\text { minimum } \cos t} \times$ 100.

From the above two ratios, we assessed how many times more does the costliest brand cost, compared to the cheapest brand. All detailed data was entered in MS excel and values expressed in counts, percentages and ratios.

\section{RESULTS}

The cost of 23 commonly used oral anti-diabetic drugs (15 single and 8 combination preparations) manufactured by different pharmaceutical companies were analysed.

Table 1: Cost variation of different brands of oral anti-diabetic agents.

\begin{tabular}{|c|c|c|c|c|c|c|c|c|}
\hline $\begin{array}{l}\text { S. } \\
\text { No. }\end{array}$ & Drugs & $\begin{array}{l}\text { Strength } \\
(\mathrm{mg})\end{array}$ & $\begin{array}{l}\text { Dosage } \\
\text { form } \\
\text { (10 tabs) }\end{array}$ & $\begin{array}{l}\text { Number of } \\
\text { brands }\end{array}$ & $\begin{array}{l}\text { Lowest } \\
\text { cost } \\
\text { (INR) }\end{array}$ & $\begin{array}{l}\text { Highest } \\
\text { cost } \\
\text { (INR) }\end{array}$ & $\begin{array}{l}\text { Cost } \\
\text { ratio }\end{array}$ & $\begin{array}{l}\text { Cost variation } \\
(\%)\end{array}$ \\
\hline \multirow{4}{*}{1.} & \multirow{4}{*}{ Glimepiride } & 1 & $\mathrm{Tab}$ & 108 & 5.9 & 212.59 & 36.032 & 3503.22 \\
\hline & & 2 & $\mathrm{Tab}$ & 108 & 4.5 & 117.90 & 26.20 & 2520 \\
\hline & & 3 & $\mathrm{Tab}$ & 27 & 31.25 & 150.64 & 4.82 & 382.05 \\
\hline & & 4 & $\mathrm{Tab}$ & 39 & 38.10 & 178.4 & 4.68 & 368.24 \\
\hline \multirow{4}{*}{2.} & \multirow{4}{*}{ Gliclazide } & 30 & $\mathrm{Tab}$ & 8 & 48.9 & 90 & 1.84 & 84.05 \\
\hline & & 40 & $\mathrm{Tab}$ & 20 & 15 & 51.5 & 3.43 & 243.33 \\
\hline & & 60 & $\mathrm{Tab}$ & 10 & 73.9 & 126.35 & 1.70 & 70.97 \\
\hline & & 80 & $\mathrm{Tab}$ & 31 & 25.33 & 82 & 3.23 & 223.73 \\
\hline \multirow{3}{*}{3.} & \multirow{3}{*}{ Glipizide } & 2.5 & $\mathrm{Tab}$ & 4 & 2.52 & 4.15 & 1.64 & 64.68 \\
\hline & & 5 & $\mathrm{Tab}$ & 11 & 1.43 & 29.47 & 20.60 & 1960.84 \\
\hline & & 10 & $\mathrm{Tab}$ & 7 & 7.2 & 25 & 3.47 & 247.22 \\
\hline \multirow{2}{*}{4.} & \multirow{2}{*}{ Glibenclamide } & 2.5 & $\mathrm{Tab}$ & 5 & 4.35 & 12.22 & 2.80 & 180.92 \\
\hline & & 5 & $\mathrm{Tab}$ & 14 & 2.66 & 50 & 18.79 & 1779.69 \\
\hline \multirow{5}{*}{5.} & \multirow{5}{*}{ Metformin } & 500 & $\mathrm{Tab}$ & 64 & 4.4 & 80 & 18.18 & 1718.18 \\
\hline & & 500 & SR & 73 & 7.43 & 280 & 37.68 & 3668.51 \\
\hline & & 850 & $\mathrm{Tab}$ & 14 & 7.18 & 38 & 5.29 & 429.25 \\
\hline & & 850 & SR & 8 & 20 & 43.8 & 2.19 & 119 \\
\hline & & 1000 & Tab & 27 & 14.6 & 48 & 3.28 & 228.77 \\
\hline
\end{tabular}




\begin{tabular}{|c|c|c|c|c|c|c|c|c|}
\hline $\begin{array}{l}\text { S. } \\
\text { No. }\end{array}$ & Drugs & $\begin{array}{l}\text { Strength } \\
(\mathrm{mg})\end{array}$ & $\begin{array}{l}\text { Dosage } \\
\text { form } \\
\text { (10 tabs) }\end{array}$ & $\begin{array}{l}\text { Number of } \\
\text { brands }\end{array}$ & $\begin{array}{l}\text { Lowest } \\
\text { cost } \\
\text { (INR) }\end{array}$ & $\begin{array}{l}\text { Highest } \\
\text { cost } \\
\text { (INR) }\end{array}$ & $\begin{array}{l}\text { Cost } \\
\text { ratio }\end{array}$ & $\begin{array}{l}\text { Cost variation } \\
(\%)\end{array}$ \\
\hline & & 1000 & SR & 59 & 10 & 61.15 & 6.11 & 511.50 \\
\hline 6. & Nateglinide & 60 & Tab & 3 & 30.76 & 94 & 3.05 & 205.59 \\
\hline \multirow{3}{*}{7.} & \multirow{3}{*}{ Repaglinide } & 0.5 & Tab & 7 & 19.90 & 88.7 & 4.45 & 345.73 \\
\hline & & 1 & Tab & 6 & 39.90 & 175.1 & 4.38 & 338.85 \\
\hline & & 2 & Tab & 8 & 75 & 231.65 & 3.08 & 208.87 \\
\hline \multirow{2}{*}{8.} & \multirow[t]{2}{*}{ Acarbose } & 25 & Tab & 7 & 47.25 & 88 & 1.86 & 86.24 \\
\hline & & 50 & Tab & 9 & 31.85 & 154 & 4.835165 & 383.52 \\
\hline \multirow{2}{*}{9.} & \multirow{2}{*}{ Voglibose } & 0.2 & Tab & 101 & 15.62 & 251.11 & 16.07 & 1507.62 \\
\hline & & 0.3 & Tab & 101 & 18.21 & 143 & 7.85 & 685.28 \\
\hline \multirow{2}{*}{10.} & \multirow{2}{*}{ Miglitol } & 25 & Tab & 4 & 48 & 146.67 & 3.05 & 205.56 \\
\hline & & 50 & Tab & 4 & 102.87 & 244.67 & 2.37 & 137.84 \\
\hline 11. & Teneligliptin & 20 & Tab & 118 & 32.9 & 210 & 6.38 & 538.30 \\
\hline 12. & Linagliptin & 5 & Tab & 2 & 247.5 & 515 & 2.08 & 108.08 \\
\hline 13. & Vildagliptin & 50 & Tab & 79 & 29 & 284 & 9.79 & 879.31 \\
\hline \multirow{2}{*}{14.} & \multirow{2}{*}{ Sitagliptin } & 50 & Tab & 3 & 140 & 414.28 & 2.95 & 195.91 \\
\hline & & 100 & Tab & 4 & 254.28 & 450 & 1.76 & 76.97 \\
\hline \multirow{3}{*}{15.} & \multirow{3}{*}{ Pioglitazone } & 7.5 & Tab & 10 & 29.7 & 77.31 & 2.60 & 160.30 \\
\hline & & 15 & Tab & 45 & 12.5 & 135 & 10.80 & 980 \\
\hline & & 30 & Tab & 29 & 20 & 185 & 9.25 & 825 \\
\hline
\end{tabular}

Table 2: Cost variation of fixed drug combinations of oral anti-diabetic agents.

\begin{tabular}{|c|c|c|c|c|c|c|c|c|}
\hline $\begin{array}{l}\text { S. } \\
\text { No. }\end{array}$ & $\begin{array}{l}\text { Fixed } \\
\text { drug } \\
\text { combination }\end{array}$ & $\begin{array}{l}\text { Strength } \\
(\mathrm{mg})\end{array}$ & $\begin{array}{l}\text { Dosage } \\
\text { form } \\
(10 \text { tabs })\end{array}$ & $\begin{array}{l}\text { Number of } \\
\text { brands }\end{array}$ & $\begin{array}{l}\text { Lowest } \\
\text { price } \\
\text { (INR) }\end{array}$ & $\begin{array}{l}\text { Highest } \\
\text { price } \\
\text { (INR) }\end{array}$ & $\begin{array}{l}\text { Cost } \\
\text { ratio }\end{array}$ & $\begin{array}{l}\text { Cost variation } \\
(\%)\end{array}$ \\
\hline \multirow{8}{*}{1.} & \multirow{8}{*}{$\begin{array}{l}\text { Glimepiride+ } \\
\text { metformin }\end{array}$} & $1+500$ & Tab & 168 & 9 & 111.65 & 12.40 & 1140.55 \\
\hline & & $1+500$ & SR & 32 & 11.2 & 98.4 & 8.78 & 778.57 \\
\hline & & $1+1000$ & Tab & 82 & 32.8 & 131.8 & 4.01 & 301.82 \\
\hline & & $1+1000$ & SR & 14 & 31.9 & 143.33 & 4.49 & 349.31 \\
\hline & & $2+500$ & Tab & 166 & 10.77 & 175.2 & 16.26 & 1526.74 \\
\hline & & $2+500$ & SR & 29 & 18.68 & 235.94 & 12.63 & 1163.06 \\
\hline & & $2+1000$ & Tab & 97 & 15.3 & 429 & 28.03 & 2703.92 \\
\hline & & $2+1000$ & SR & 16 & 33.90 & 159.50 & 4.70 & 370.50 \\
\hline \multirow{5}{*}{2.} & \multirow{5}{*}{$\begin{array}{l}\text { Glimepiride+ } \\
\text { metformin+ } \\
\text { pioglitazone }\end{array}$} & $1+500+7.5$ & Tab & 20 & 25 & 96.25 & 3.85 & 285 \\
\hline & & $1+500+15$ & Tab & 96 & 20 & 167 & 8.35 & 735 \\
\hline & & $2+500+7.5$ & Tab & 26 & 27.3 & 122.7 & 4.49 & 349.45 \\
\hline & & $2+500+15$ & Tab & 101 & 20 & 202 & 10.10 & 910 \\
\hline & & $2+500+30$ & Tab & 3 & 89 & 121.25 & 1.36 & 36.23 \\
\hline \multirow{4}{*}{3.} & \multirow{4}{*}{$\begin{array}{l}\text { Voglibose+ } \\
\text { metformin+ } \\
\text { glimepiride }\end{array}$} & $0.2+500+1$ & Tab & 100 & 20 & 202.5 & 10.12 & 912.50 \\
\hline & & $0.2+500+2$ & Tab & 101 & 20 & 224.66 & 11.23 & 1023.30 \\
\hline & & $0.3+500+1$ & Tab & 38 & 88.50 & 210 & 2.37 & 137.28 \\
\hline & & $0.3+500+2$ & Tab & 44 & 80 & 260 & 3.25 & 225 \\
\hline \multirow{2}{*}{4.} & \multirow{2}{*}{$\begin{array}{l}\text { Gliclazide+ } \\
\text { metformin }\end{array}$} & $60+500$ & Tab & 19 & 43 & 145 & 3.37 & 237.20 \\
\hline & & $80+500$ & Tab & 86 & 26.9 & 500 & 18.58 & 1758.73 \\
\hline 5. & $\begin{array}{l}\text { Glipizide+ } \\
\text { metformin }\end{array}$ & $5+500$ & Tab & 17 & 6.72 & 140.52 & 20.91 & 1991.07 \\
\hline \multirow{2}{*}{6.} & \multirow{2}{*}{$\begin{array}{l}\text { Teneligliptin+ } \\
\text { metformin }\end{array}$} & $20+500$ & Tab & 115 & 47.17 & 163.33 & 3.46 & 246.25 \\
\hline & & $2+1000$ & Tab & 80 & 50.62 & 170 & 3.35 & 235.83 \\
\hline \multirow{2}{*}{7.} & \multirow{2}{*}{$\begin{array}{l}\text { Voglibose+ } \\
\text { metformin }\end{array}$} & $0.2+500$ & Tab & 85 & 30.90 & 283.87 & 9.18 & 818.67 \\
\hline & & $0.3+500$ & Tab & 59 & 34 & 318 & 9.35 & 835.29 \\
\hline \multirow{2}{*}{8.} & \multirow{2}{*}{$\begin{array}{l}\text { Repaglinide+ } \\
\text { voglibose }\end{array}$} & $1+0.2$ & Tab & 4 & 128 & 165 & 1.28 & 28.90 \\
\hline & & $1+0.3$ & Tab & 5 & 131 & 165 & 1.25 & 25.95 \\
\hline
\end{tabular}


Table 3: Ceiling price of oral anti-diabetic drugs under price control by NPPA, GOI.

\begin{tabular}{|c|c|c|c|c|c|}
\hline $\begin{array}{l}\text { S. } \\
\text { No. }\end{array}$ & Drugs & $\begin{array}{l}\text { Strength } \\
\text { (mg) }\end{array}$ & Dosage form & Number of tablets & Ceiling price (INR/unit) \\
\hline \multirow{2}{*}{1.} & \multirow{2}{*}{ Glimepiride } & 1 & $\mathrm{Tab}$ & 1 & 3.60 \\
\hline & & 2 & Tab & 1 & 5.72 \\
\hline \multirow{6}{*}{2.} & \multirow{6}{*}{ Metformin } & 500 & Tab & 1 & 1.51 \\
\hline & & 500 & SR & 1 & 1.92 \\
\hline & & 750 & Tab & 1 & 3.05 \\
\hline & & 750 & SR & 1 & 2.40 \\
\hline & & 1000 & Tab & 1 & 3.61 \\
\hline & & 1000 & SR & 1 & 3.66 \\
\hline
\end{tabular}

\section{Single drug preparations}

Among single drug preparations, a total 15 commonly used preparations were analysed (Table 1). The highest percentage cost variation was found for metformin $500 \mathrm{mg}$ sustained release $(3668 \%)$. Other significance high cost variations were glimepiride $1 \mathrm{mg}(3503 \%)$, gimipiride 2 $\mathrm{mg}(2520 \%)$, glipizide $5 \mathrm{mg}$ (1960\%), glibenclamide $5 \mathrm{mg}$ $(1779 \%)$ and metformin $500 \mathrm{mg}(1718 \%)$. The minimum cost variation was found with glipizide $2.5 \mathrm{mg}(65 \%)$ followed by gliclazide $60 \mathrm{mg}(71 \%)$, sitagliptin $100 \mathrm{mg}$ (77\%), gliclazide $30 \mathrm{mg}(84 \%)$ and acarbose $25 \mathrm{mg}(86 \%)$.

Maximum number of brands is available for glimepiride 1 $\mathrm{mg}$ and $2 \mathrm{mg}$ (108 brands each) followed by teneligliptin $20 \mathrm{mg}$ (118 brands), voglibose $0.2 \mathrm{mg}$ and $0.3 \mathrm{mg}$ (101 brands). Minimum number of brands were available for linagliptin $5 \mathrm{mg}$ (2 brands) followed by sitagliptin $50 \mathrm{mg}$ and nateglinide $60 \mathrm{mg}$ ( 3 brands each). The most expensive was sitagliptin $100 \mathrm{mg}$ (₹ 450) followed by sitagliptin 50 mg (₹ 414) and metformin 500 mg SR (₹ 280). Minimum cost was of glipizide $5 \mathrm{mg}$ (₹ 1.43 ) followed by glipizide $2.5 \mathrm{mg}$ (₹ 2.52) and gliclazide $5 \mathrm{mg}$ (₹ 2.66).

\section{Fixed dose combinations}

Amongst fixed dose combinations, a total of 8 commonly used combination preparations were analyzed (Table 2). The maximum cost variation was found for glimepiride 2 mg+metformin $1000 \mathrm{mg}$ (2703\%). Other significant high cost variations were glipizide $5 \mathrm{mg}+$ metformin $500 \mathrm{mg}$ (1991\%), gliclazide $80 \mathrm{mg}+$ metformin $500 \mathrm{mg}$ (1758\%), glimepiride $2 \mathrm{mg}+$ metformin $500 \mathrm{mg}$ (1527\%) and glimepiride $2 \mathrm{mg}+$ metformin $500 \mathrm{mg}$ SR (1163\%). While minimum cost variation was found in repaglinide 1 mg+voglibose $0.3 \mathrm{mg}(26 \%)$ followed by repaglinide 1 $\mathrm{mg}+$ voglibose $0.2 \mathrm{mg}$ (29\%), glimepiride $2 \mathrm{mg}+$ metformin $500 \mathrm{mg}$ +pioglitazone $30 \mathrm{mg}(36 \%)$, voglibose 0.3 $\mathrm{mg}+$ metformin $500 \mathrm{mg}+$ glimepiride $1 \mathrm{mg}$ (137\%) and voglibose $0.3 \mathrm{mg}+$ metformin $500 \mathrm{mg}+$ glimepiride $2 \mathrm{mg}$ $(225 \%)$.

Maximum number of brands were available for glimepiride $1 \mathrm{mg}+$ metformin $500 \mathrm{mg}$ (168 brands) followed by glimepiride $2 \mathrm{mg}+$ metformin $500 \mathrm{mg}+$ pioglitazone $15 \mathrm{mg}$ and voglibose $0.2 \mathrm{mg}+$ metformin $500 \mathrm{mg}+$ glimepiride 2 mg (101 brands each). Minimum number of brands were available for glimepiride 2 mg+metformin 500 $\mathrm{mg}$ +pioglitazone $30 \mathrm{mg}$ ( 3 brands) followed by repaglinide $1 \mathrm{mg}+$ voglibose $0.2 \mathrm{mg}$ (4 brands). Maximum price was of gliclazide $80 \mathrm{mg}+$ metformin $500 \mathrm{mg}$ (₹ 500) followed by voglibose $0.3 \mathrm{mg}+$ metformin $500 \mathrm{mg}$ (₹ 318) and voglibose $0.3 \mathrm{mg}+$ metformin $500 \mathrm{mg}+$ glimepiride $2 \mathrm{mg}$ (₹ 260). Minimum cost was of glipizide $5 \mathrm{mg}+$ metformin 500 $\mathrm{mg}$ (₹ 6.72) followed by glimepiride $1 \mathrm{mg}+$ metformin 500 mg (₹ 9) and glimepiride $2 \mathrm{mg}+$ metformin $500 \mathrm{mg}$ (₹ 10.77).

\section{DISCUSSION}

In the present study, a noticeable cost variation was found in different brands of the same oral antidiabetic drug. The most commonly prescribed single drug therapy was metformin $500 \mathrm{mg} \mathrm{SR}{ }^{13}$ It showed the maximum price variation of $3668 \%$. This was unlike the study by Dharani et al and Mehani et al wherein the maximum cost variation was seen with glimepiride $2 \mathrm{mg}$ and $1 \mathrm{mg}$ respectively. ${ }^{14,15}$ The highest cost variation among multiple drug therapy was seen with the combination glimepiride 2 mg+metformin $1000 \mathrm{mg}(2703 \%)$ unlike the study by Dharani et al and Mehani et al in which maximum cost variation was seen with glimepiride $1 \mathrm{mg}+$ metformin 500 mg. ${ }^{14,15}$

Drug prices in the Indian market are controlled by the NPPA of the Government of India. It fixes the ceiling price of a particular drug based on its essentiality. This prevents pharmaceutical companies from selling medicines from the drugs prices control order (DPCO) list at a price which is higher than the fixed price. ${ }^{16}$ Amongst various oral antidiabetic drugs, only two drugs namely glimepiride and metformin are under DPCO price control (Table 3). Other newer agents frequently prescribed by the treating physician, either alone or in combination, are not under price control. Wealth is not distributed equally. So, the gap between the rich and poor continues to widen in India. ${ }^{17}$ The poor and middle classes cannot afford high quality health care like the wealthy. ${ }^{18}$ Hence, prescribing doctors should strongly consider drug prices especially in a 
developing country like India, where a majority patients are paying out of their pockets for their medical expenses and are not covered by insurance schemes. ${ }^{8}$ In India, more than $80 \%$ health financing is borne by patients. If an expensive brand is prescribed, patients have to unnecessarily pay more for the same medicine which in turn increases the economic burden on them. ${ }^{8}$ This can be prevented by educating the masses about various health insurance facilities available, so that good quality and affordable healthcare can be given to them.

Being a chronic disease, the management of DM requires continuous medical care. Prescribing doctors may not necessarily know the cost of the drugs. Some clinicians also get lured by various incentives provided by the pharmaceutical companies and thus prescribe more expensive drugs when a cheaper alternative is available. Rational use of drugs is defined as patients receiving medications appropriate to their clinical needs, in doses that meet their own individual requirements, for an adequate period of time and at the lowest possible cost. ${ }^{17}$ Many brands of a particular drug available for an indication makes the decision complex and less rational. Thus the physician's P drug list should consist of selected safe, efficacious and cost-effective drugs to ensure their rational use.

\section{CONCLUSION}

The present study shows that there is a wide variation in the cost of different brands of the same oral anti-diabetic drug currently available in Indian market. Pharmacoeconomics should be given greater emphasis during medical training. Physicians should switch to cost effective therapy and prescribe rationally. Education about health insurance facility should be given to all people. More oral anti-diabetic drugs should be covered under DPCO so that all patients irrespective of their financial condition will be able to afford these medicines. In case of non-compliance to DPCO by pharmaceutical companies, more stringent actions should be ensured.

\section{Funding: No funding sources}

Conflict of interest: None declared

Ethical approval: The study was approved by the Institutional Ethics Committee

\section{REFERENCES}

1. Kruger D, Marcus AO. Psychological motivation and patient education: a role for continuous glucose monitoring. Diabetes Technol Ther. 2000;2:93.

2. Tripathi BR, Srivastava AK. Diabetes mellitus: Complications and therapeutics. Med Sci Monit. 2006;12(7):130-47.

3. Saeedi P, Petersohn I, Salpea P, Malanda B, Karuranga S, Unwin N, et al. Global and regional diabetes prevalence estimates for 2019 and projections for 2030 and 2045: Results from the International Diabetes Federation Diabetes Atlas, 9th edition. Diabetes Res Clin Prac. 2019;1:107843.

4. Atre S. Addressing policy needs for prevention and control of type 2 diabetes in India. Perspect Public Health. 2015;135(5):257-63.

5. Joshi SR, Parikh RM. India-diabetes capital of the world: now heading towards hypertension. J Assoc Physicians India. 2007;55:323-4.

6. Piette JD, Heisler M, Wagner TH. Cost-related medication underuse: do patients with chronic illnesses tell their doctors? Arch Intern Med. 2004;164(16):1749-55.

7. Thomas M. Rational drug use and essential drug concept. In: Parthasarthi G, Nyfort-Hasen K, eds. A Textbook of Clinical Pharmacy Practice. 1st ed. Hyderabad: Orient Longman; 2004: 72-3.

8. Allan GM, Lexchin J, Wiebe N. Physician awareness of drug cost: a systematic review. PLoS Med. 2007;4(9):283.

9. Cox ER, Jernigan C, Coons SJ, Draugalis JL. Medicare beneficiaries' management of capped prescription benefits. Med Care. 2001;39(3):296-301.

10. Goldman DP, Joyce GF, Escarce JJ, Pace JE, Solomon MD, Laouri M, et al. Pharmacy benefits and the use of drugs by the chronically ill. JAMA. 2004;291(19):2344-50.

11. Jadhav NB, Bhosale MS, Adhav CV. Cost analysis of study of oral antidiabetic drugs available in Indian market. Int J Med Res Health Sci. 2013;2:63-9.

12. American Diabetes Association. Standards of Medical Care in Diabetes. Am Diabetes Association Diabet Care. 2014;37(1):14-80.

13. Khan GH, Aqil M, Pillai KK, Ahmad MA, Kapur P, Ain MA, et al. Therapeutic adherence: A prospective drug utilization study of oral hypoglycemic in patients with type 2 diabetes mellitus. Asian Pac J Trop Dis. 2014;4(1):347-52.

14. Dharani D, Rajesh B, Shashirekha CH. Cost analysis study of commonly used oral hypoglycaemic agents available in Indiaa pharmacoeconomic study. Indian $\mathbf{J}$ Pharm Pharmacol. 2017;4;22-6.

15. Mehani R, Sharma P. Cost variation analysis of oral anti-diabetic drugs. Int J Basic Clin Pharmacol. 2018;7(9):1709-14.

16. Das SC, Mandal M, Mandal SC. A critical study on availability and price variation between different brands: Impact on access to medicines. Indian J Pharm Sci. 2007;69(1):160-3.

17. Rational use of drug. World Health Organization. [Last cited on 2012 Jan 02]. Available from: http://www.whoindia.org/LinkFiles/GPP_Rational_U se_of_Medicines.pdf .

18. Baru M, Acharya A, Acharya S, Kumar AS, Nagraj K. Inequities in access to health services in india: caste, class and region. Econ Polit Wkly. 2010;45:49-58.

Cite this article as: Gupta KS, Pardeshi ML, Hiray RS. Cost variation analysis of commonly prescribed anti-diabetic drugs available in Indian market: a pharmaco-economic study. Int J Basic Clin Pharmacol 2022;11:47-51. 